\title{
Magnetic shielding using high-temperature superconductors
}

\author{
V GANESAN, R SRINIVASAN, S ASWATHY\#, \\ K D CHANDRASEKARAN ${ }^{+}$, B SRINIVAS $^{+}, \mathrm{U} \mathrm{V}$ VARADA RAJU ${ }^{+}$, \\ G V SUBBA RAO ${ }^{\$}$, R GOPALAN $^{(a)}$ and T RAJASEKARAN ${ }^{(a)}$ \\ Inter University Consortium for DAE Facilities, University Campus, Khandwa Road, Indore \\ 452001 , India \\ "Department of Physics, Indian Institute of Technology, Madras 600036, India \\ + Materials Science Research Centre, Indian Institute of Technology, Madras 600036 , India \\ 'Central Electrochemical Research Institute, Karaikudi 623006, India \\ (") Defence Metallurgical Research Laboratory, Hyderabad 500258, India
}

MS received 21 May 1993; revised 29 October 1993

\begin{abstract}
Magnetic shields of various high-temperature superconductors, $\mathrm{YBa}_{2} \mathrm{Cu}_{3} \mathrm{O}_{7-x}$ (YBCO), $\mathrm{YBa}_{2} \mathrm{Cu}_{3} \mathrm{O}_{7-x}-\mathrm{Ag}$ composites (random inclusions as well as non-random coatings) and $\mathrm{Bi}_{2} \mathrm{Sr}_{2} \mathrm{Ca}_{2} \mathrm{Cu}_{3} \mathrm{O}_{x}$ (BSCCO) were prepared by uniaxial as well as isostatic compression with various dimensions. The shielding properties were measured at $77 \mathrm{~K}$ for dc and ac magnetic fields in the range of frequencies from $100 \mathrm{~Hz}$ to $10 \mathrm{kHz}$. The critical penetration field (CPF), defined as the value of the applied magnetic field at which a detectable field was observed inside the cylinder, varied from cylinder to cylinder and also with the ageing of the cylinders in the case of YBCO shields. The highest value of CPF was $16 \mathrm{G}$ at $77 \mathrm{~K}$ for YBCO shield prepared by isostatic compression. Even though the stability of BSCCO shields with respect to ageing is good, the CPF values are very low compared to those for YBCO. Detailed studies were performed in the case of YBCO shields. The CPF decreased as a function of time over a period of $\mathbf{9 0}$ days. The CPF decreased as the frequency of the applied field was increased. The wave form of the field inside the pot for a sinusoidal applied field was highly distorted and showed the presence of higher harmonics with appreciable amplitude. The wave form was Fourier-analysed to yield the field inside the shield along with the harmonics. The shields with $\mathrm{Ag}$ addition seem to give better performance at high fields.
\end{abstract}

Keywords. High-temperature superconductors; magnetic shielding; $\mathrm{YBa}_{2} \mathrm{Cu}_{3} \mathrm{O}_{7-x}$; $\mathrm{Bi}_{2} \mathrm{Sr}_{2} \mathrm{Ca}_{2} \mathrm{Cu}_{3} \mathrm{O}_{x} ; \mathrm{YBCO}-\mathrm{Ag}$ composites.

\section{Introduction}

Electromagnetic shielding is one of the subjects of interest in which the high- $T_{c}$ superconductors can be put into application. Many researchers all over the world started working in this area of high- $T_{c}$ materials because this is likely to be commercialized soon. Magnetic shielding is crucial to many advanced technological applications. This plays a vital role in medical industry, physics research and the electronic industry. For example, with the discovery of SQUIDs it is now possible to detect very small changes in magnetic fields as in biomagnetic applications. These fields are of the order of few picogauss and are of low frequency of a few hertz. When compared to even the earth's field these are smaller by several orders and demand a very effective shielding of the order of $120-200 \mathrm{~dB}$ for any useful application.

It also finds applications in high-energy physics for providing a field-free region for the charged particles as well as for shielding human beings and other sensitive instruments against steady as well as pulsed fields. There is no need to say that sophisticated electronic instrument calibrations require electromagnetic shielding of 
very high order. The earth is not the only source of magnetic field. Power lines and EM waves from different transmitters etc. are also sources of disturbances. A good review on the importance of shielding can be had from Newman (1990). Conventional high-sensitivity magnetometers and SQUIDs for physics as well as for biomagnetic applications use $\mathrm{Nb}$ shields normally.

Metals with high conductivity, like $\mathrm{Cu}$, are good shields as they can shield highfrequency fields to the required levels and also they are cheaper and economical compared to superconductors, which require a cryogen for cooling. But metals are ineffective in providing shielding against low-frequency fields and dc fields. Only superconductors are the solution for this. Advent of high- $T_{c}$ materials brought down the cost because they can use a comparatively cheaper cryogen like liquid nitrogen. Also, these materials, even though they have low $J_{c}$ values, have fairly good shielding properties.

Magnetic shielding using high- $T_{c}$ materials was extensively studied by various groups (Hattori et al 1988; Aswathi et al 1989; Cui et al 1989; Purpura and Clem 1989; Shigematsu et al 1989; Symko et al 1989; Willis et al 1989; Vlakhov et al 1991, etc for YBCO cylinders; Karthikeyan et al 1989, etc for plasma spray coated films; and Russell and Taylor 1989, etc for discs). For useful applications studies on closed enclosures are essential. In this paper we report the fabrication of shields with high- $T_{c}$ materials and an outline of the results.

\section{Experimental}

Details of the experimental arrangement can be had from Ganesan and Srinivasan (1989). It consists of a long solenoid producing an axial magnetic field, at the centre of which is placed the shield of the superconductor. The solenoids were calibrated using a flux meter (Siemens). The shield was closed with a lid of the same material to provide a totally enclosed region for study. A search coil with an effective area of $2121 \times 10^{-4} \mathrm{~m}^{2}$ was placed coaxially at the centre of the cylindrical pot. For dc measurements the search coil was connected to the flux meter. The smallest detectable field in this arrangement was $50 \mathrm{mG}$. For ac measurements the search coil was connected to a Tektronix dual trace oscilloscope. The smallest detectable field for ac measurements is determined by noise in the circuit and was $100 \mathrm{mG}$ at $1 \mathrm{kHz}$.

The critical penetration field (CPF) is defined as the minimum value of the applied field at which a detectable signal is seen from the search coil. In dc measurements, the current through the solenoid was slowly increased till the flux meter showed a non-zero reading. In ac measurements the amplitude of the ac current through the solenoid was varied till the signal from the search coil showed a detectable emf. AC measurements were carried out in the range of frequencies from $100 \mathrm{~Hz}$ to $10 \mathrm{kHz}$. Most of the measurements were done at $77 \mathrm{~K}$ with the cylinder in a bath of liquid nitrogen. A few measurements were performed at $4.2 \mathrm{~K}$ with the cylinder immersed in liquid helium.

\subsection{Shields of YBCO prepared by axial compression}

Seven cylinders of YBCO were examined. All of them had the same dimensions of $4 \mathrm{~cm}$ outer diameter, $5 \mathrm{~cm}$ height and $1 \mathrm{~cm}$ wall thickness. These cylinders were 
prepared from YBCO powder prepared by solid state reaction technique. The powder was single-phase $\mathrm{YBa}_{2} \mathrm{Cu}_{3} \mathrm{O}_{7-y}$ as ascertained by $\mathrm{X}$-ray powder diffraction. The powder was dry-compressed axially in a die to 10 ton pressure to form a cylindrical pot. The cylinder was then kept in a furnace and its temperature was raised at the rate of $60^{\circ} \mathrm{C} / \mathrm{h}$ to $950^{\circ} \mathrm{C}$. The pot was annealed in air at this temperature for $24 \mathrm{~h}$ and slow-cooled to room temperature. A subsequent oxygen treatment was given as follows: $900^{\circ} \mathrm{C}$ for $24 \mathrm{~h}$, slow cooling to $600^{\circ} \mathrm{C}$ and maintaining at that temperature for $24 \mathrm{~h}$, and then slow cooling to room temperature in $24 \mathrm{~h}$, all the above processes being carried out in flowing oxygen. The warming and cooling rates were chosen so as to avoid cracks in the cylinder due to the differential expansion during the warming and cooling cycles. A YBCO disc of the same thickness was also prepared in the same fashion to cover the disc. The total weight of the cylinder and the disc is about $220 \mathrm{~g}$. The $\mathrm{X}$-ray powder diffractogram shows that the samples were in single phase with orthorhombic structure having unit cell dimensions $a=3.830(3) \AA, b=3.901$ (3) $\AA$, $c=11 \cdot 704(7) \AA$. A pellet of the material prepared under the same conditions showed zero resistance at $90 \mathrm{~K}$.

\subsection{Shields of YBCO prepared by isostatic compression}

YBCO shields were also prepared by isostatic compression using the facilities available at DMRL, Hyderabad. The necessary dies and rubber bags were made for this purpose. Superconducting YBCO powder was used for making the shields. Five such shields were prepared with varying wall thickness and also with flat as well as hemispherical bottom. Typical dimensions of the shields are $50 \mathrm{~mm}$ in length and $40 \mathrm{~mm}$ in outer diameter. After compression the shields were sintered in air for $24 \mathrm{~h}$ at $930^{\circ} \mathrm{C}$, with heating and cooling rates maintained at $60^{\circ} \mathrm{C} / \mathrm{h}$ to avoid cracking due to thermal contraction. These shields were then oxygen-annealed at $900^{\circ} \mathrm{C}$ for $24 \mathrm{~h}$ and at $600^{\circ} \mathrm{C}$ for another $24 \mathrm{~h}$ with the same heating and cooling rates of $60^{\circ} \mathrm{C} / \mathrm{h}$.

\subsection{Shields of $\mathrm{YBCO}$ with $\mathrm{Ag}$}

One such shield was prepared with $10 \%$ silver powder added before making the compression. Silver powder was added to the superconducting powder and was mixed thoroughly for $24 \mathrm{~h}$ in a ball mill before it was isostatically compressed. Sintering as well as oxygen annealing procedures are essentially same as above except that the maximum temperature was restricted to $850^{\circ} \mathrm{C}$.

One of the shields of YBCO was prepared by the non-random ceramic coating of the grains of YBCO by Ag. In this method each superconducting YBCO grain is coated with $\mathrm{Ag}$ by chemical process and then the bulk material was fabricated and oxygen-annealed. The details of preparation of such composites can be had from Srinivas and Subba Rao (1992).

\subsection{Shields of BSCCO prepared by isostatic compression}

Shields of BSCCO materials were prepared from the superconducting BSCCO powder prepared by nitrate precursor technique and the shields were formed by isostatic 


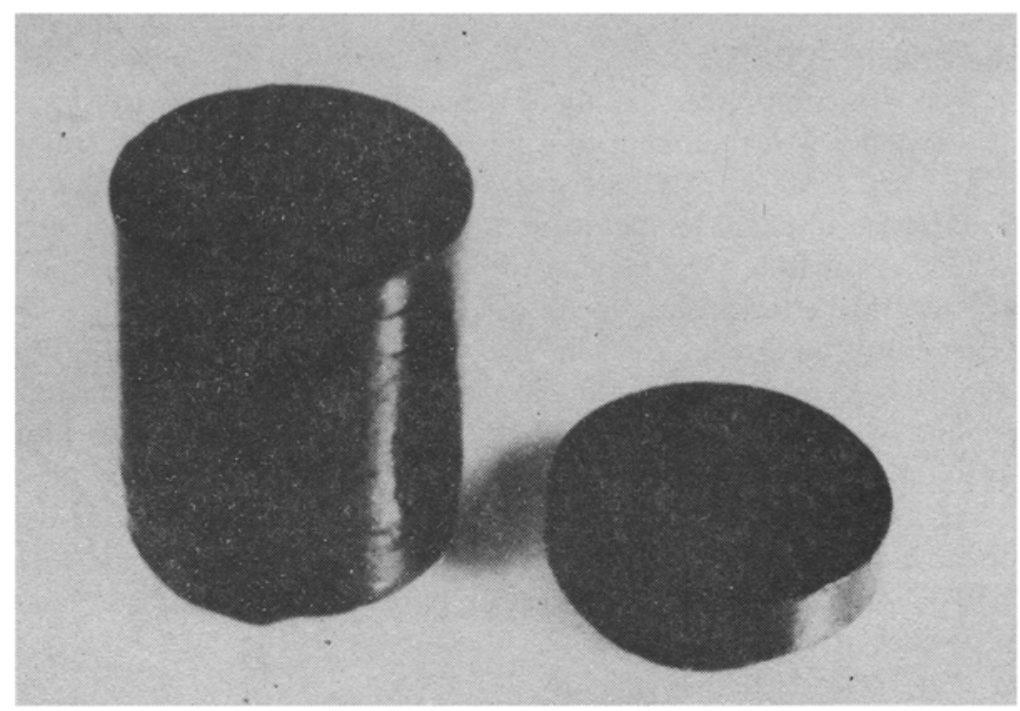

Figure 1. Photograph of the YBCO shield prepared by axial compression.

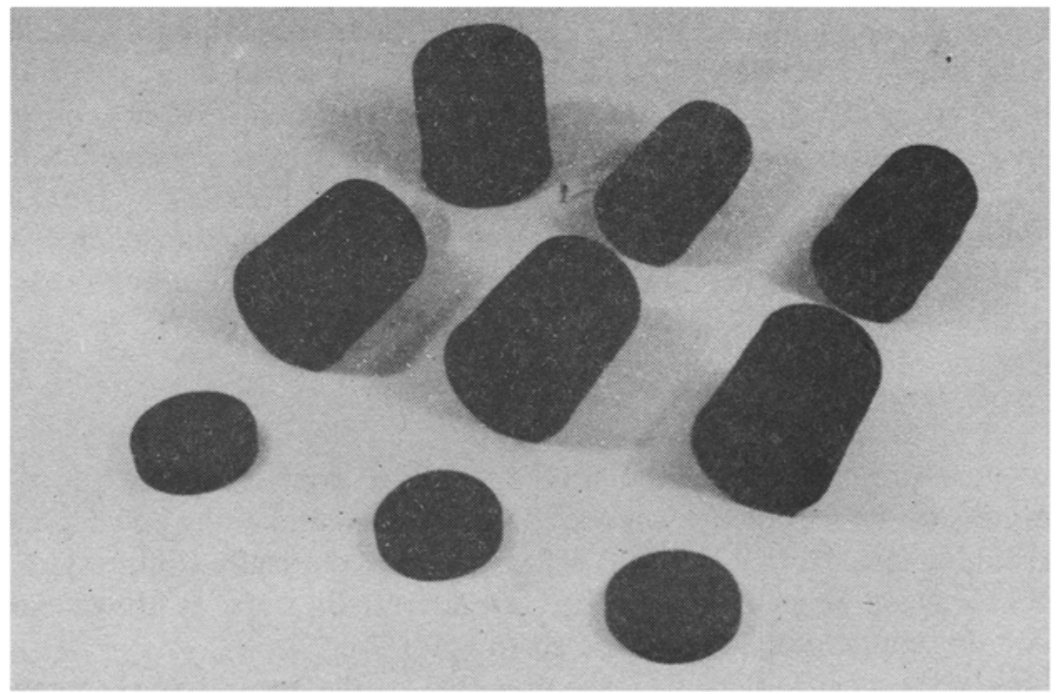

Figure 2. Photograph of the YBCO shields prepared by isostatic compression.

compression into the required shapes. Different shapes of the shields were tried to see whether it is possible to fabricate such shapes which may be useful while implementing the applications.

Figure 1 shows the photograph of one of the shields of YBCO prepared by axial compression. Figure 2 shows the YBCO shields prepared by isostatic compression. The detailed analysis of the results on these shields will be published elsewhere. This 
figure is repeated here for the sake of completeness. Figure 3 shows the BSCCO shields prepared by isostatic compression.

\section{Results and discussion}

Table 1 gives the CPF values for different shields. YBCO shields prepared by isostatic compression (iso) show improved shielding compared to the shields prepared by axial compression. The CPF of the YBCO shield (axial) was $12 \mathrm{G}$ at $77 \mathrm{~K}$ for dc field and decreases as the frequency of the applied field is increased in the case of ac fields. The CPF of the YBCO shields (iso) are $16-17 \mathrm{G}$ at $77 \mathrm{~K}$. The frequency response is of the same nature as that of axially prepared shields. The addition of $\mathrm{Ag}$ to YBCO does not improve CPF but it improves the shielding effectiveness to three times compared to the shields without Ag. However, the effect of frequency on CPF is to

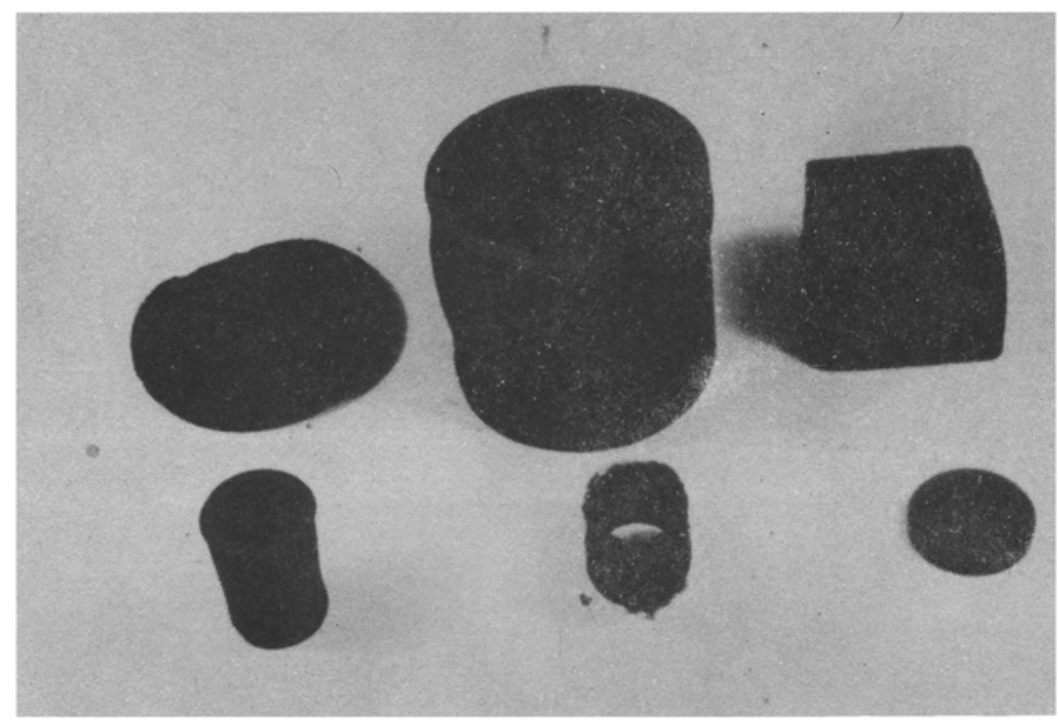

Figure 3. Photograph of the BSCCO shields prepared by isostatic compression.

Table 1. Description, wall thickness and CPF values for six high- $T_{c}$ shields."

\begin{tabular}{lcc}
\hline Description & $\begin{array}{c}\text { Wall thickness } \\
d(\mathrm{~mm})\end{array}$ & $\begin{array}{c}\text { CPF } \\
(\mathrm{G})\end{array}$ \\
\hline YBCO (axially compressed) & 10 & 12 \\
YBCO (isostatically compressed) & 10 & 12 \\
YBCO (isostatically compressed) & 4 & 15 \\
YBCO (isostatically compressed) $+10 \% \mathrm{Ag}$ & 8 & 16 \\
YBCO + Ag (non-random composite) & 6 & 5 \\
BSCCO (isostatically compressed) & 4 & 1 \\
\hline
\end{tabular}


decrease CPF more rapidly than in the case of $\mathrm{YBCO}$ without Ag. The results are in good agreement with those reported in the literature.

The field inside the shield was highly distorted showing the presence of higher harmonics with appreciable amplitude. Figure 4 shows the oscilloscope trace of the signal inside the shield along with the applied field of amplitude $44 \mathrm{G}$ at $426 \mathrm{~Hz}$. The waveform of the field inside was Fourier-analysed to yield the fundamental as well as harmonics. Figure 5 shows a sample plot of $B$ (inside) as a function of $B$ (applied) for a shield of YBCO prepared by axial compression as well as the harmonics. Such an estimate of the magnitude of the field inside the shield is very useful to understand the performance of the shield. The field inside the shield is the combination of fundamental and harmonics of the applied frequency. The estimation of the harmonics is useful to study the nonlinearity of the system and the associated hysteretic behaviour. Also the ratio of the field penetrated to the field applied gives the effectiveness of the

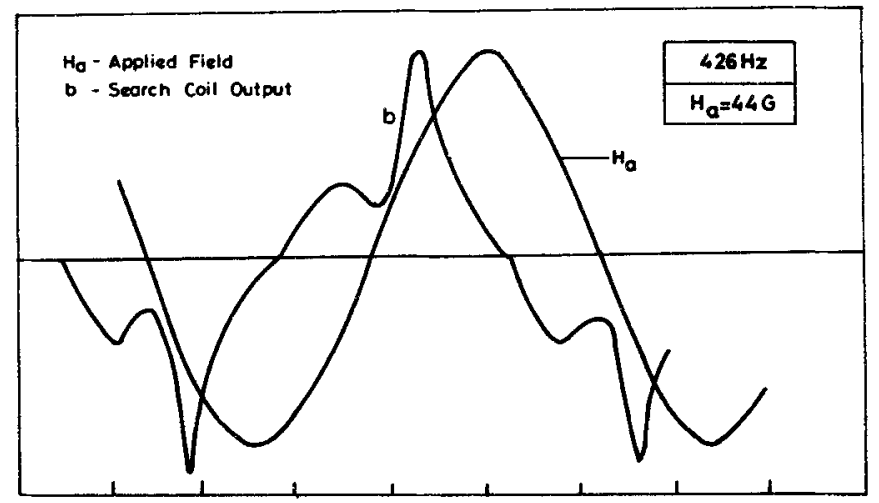

Figure 4. Oscilloscope trace of the field inside and that of the applied field for the shield of YBCO prepared by axial compression.
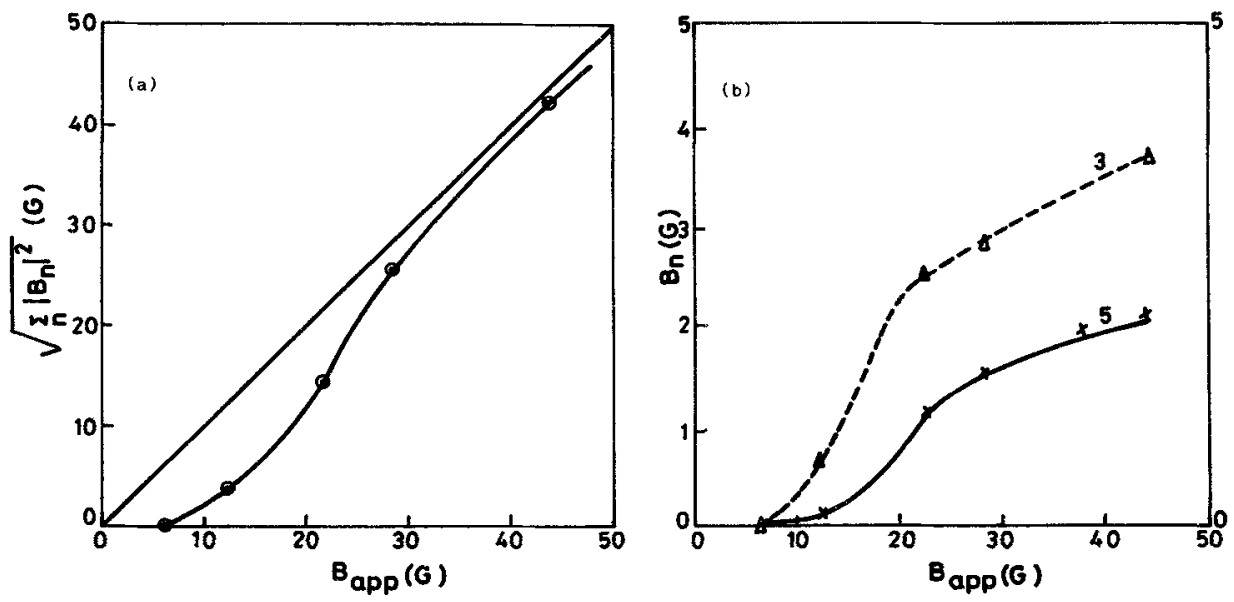

Figure 5. (a) The magnitude of the maximum magnetic induction field inside the shield as a function of the applied field. (b) Variation of the third and fifth harmonics inside the shield as a function of the applied field for the shield prepared by axial compression. 
shield. This idea of the penetrated field is very important in designing multilayer shields.

Shields of YBCO with $\mathrm{Ag}$ prepared by non-random ceramic coating method show a CPF of about $5 \mathrm{G}$ only. The different shields of BSCCO materials show CPF values in the range 1-2 $\mathrm{G}$ only. Even though the absolute value of CPF is very low compared to that for YBCO, these shields are attractive because of less degradation of shielding properties with ageing. In the case of YBCO CPF decreased from $12 \mathrm{G}$ to $2 \mathrm{G}$ in a period of 90 days. Detailed ageing studies on BSCCO shields are being carried out. It is worth mentioning here that Koike et al (1991) have developed a large magnetic shielding vessel of BSCCO (dimensions are $320 \mathrm{~mm}$ diameter, $640 \mathrm{~mm}$ depth and $25 \mathrm{~mm}$ thickness) which can provide a shielding effectiveness of $100 \mathrm{~dB}$ for weak fields.

With YBCO shields fields up to $15-20 \mathrm{G}$ can be shielded effectively. The addition of Ag does not bring about an increase in CPF. However, for the same value of CPF, the $\mathrm{YBCO}-\mathrm{Ag}$ composite has a larger shielding effectiveness than the YBCO shields for fields as large as three times CPF. The low value of CPF in these materials is due to the low value of $J_{\mathfrak{r}}$. We are trying to prepare a shield of YBCO prepared by melt-grown process so that the $J_{c}$ values can be made high. Regarding long-term stability the BSCCO shields seem to be promising provided their $J_{c}$ values can be increased. In conclusion, magnetic shields can be fabricated out of high- $T_{c}$ materials which can provide a shielding effectiveness of $60-100 \mathrm{~dB}$ at low frequencies over a volume of about $30-40 \mathrm{~cm}^{3}$. Fabrication of large shields or even shielded rooms for neuromagnetic measurements may be the immediate future outcome of the application of high- $T_{c}$ materiais.

\section{Acknowledgements}

This work was carried out through a grant sanctioned by the Program Management Board on Superconductivity, Government of India. This is gratefully acknowledged. The authors thank Prof. G Rangarajan of the Low Temperature Lab., Indian Institute of Technology, Madras, for his encouragement.

\section{References}

Aswathy S, Ganesan V, Chandrasekaran K D, Subba Rao G V and Srinivasan R 1989 DAE Symposium on Solid State Physics, Madras, India

Cui C J, Wang S G, Jiang H M, Li J Z, Lin C D, Liu H Z, Zheng Q L, Fu Y S, Luo Z L and Qiao W C 1989 IEEE Trans. Mag. 252273

Ganesan V and Srinivasan R 1989 High Temperature Superconductors, IIT Madras Newsletter 21

Hattori T, Higemoto N, Kanazawa S and Kobayashi M 1988 Jpn. J. Appl. Phys. 27 L1120

Karthikeyan J, Paithankar A S, Sreekumar K P, Venkataramani N and Rohatgi V K 1989 Cryogenics 29915

Koike A, Hoshino K, Ohta H, Kotaka H, Sudoh E, Katoh K and Takahara H 1991 Physica C185-1892613

Newinan D 1990 Superconductor Ind. Fall 16

Purpura J W and Clem T R 1989 IEEE Trans. Mag. 252506

Russel G J and Tayler K N R 1989 J. Appl. Phys. 665498

Shigematsu K, Ohta H, Hoshino K, Takayama H, Yagishita O, Yamazaki S, Takahara H and Aono M 1989 Jpn. J. Appl. Phys. $28 \mathrm{L813}$

Srinivas B and Subba Rao G V 1992 Bull. Mater. Sci. 15403

Symko O G, Yeh W J, Zheng D J and Kulkarni S 1989 J. Appl. Phys. D65 2142

Vlakhov E S, Kovachev V T, Polak M, Majoros M, Dimitriev Y B, Jambazov S, Kashchieva E and Staneva A 1991 Physica C175 335

Willis J O, McHenry M E, Maley M P and Sheinberg H 1989 IEEE Trans. Mag. 252502 